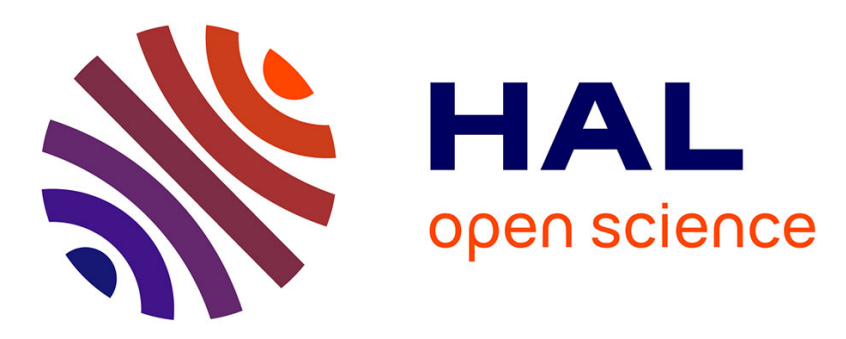

\title{
Focusing of Rayleigh waves with gradient-index phononic crystals
}

Jinfeng Zhao, Bernard Bonello, Loic Becerra, Olga Boyko, Rémi Marchal

\section{To cite this version:}

Jinfeng Zhao, Bernard Bonello, Loic Becerra, Olga Boyko, Rémi Marchal. Focusing of Rayleigh waves with gradient-index phononic crystals. Applied Physics Letters, 2016, 108, pp.221905 - 53504 . 10.1063/1.4953168. hal-01414717

\section{HAL Id: hal-01414717 https://hal.sorbonne-universite.fr/hal-01414717}

Submitted on 15 Dec 2016

HAL is a multi-disciplinary open access archive for the deposit and dissemination of scientific research documents, whether they are published or not. The documents may come from teaching and research institutions in France or abroad, or from public or private research centers.
L'archive ouverte pluridisciplinaire HAL, est destinée au dépôt et à la diffusion de documents scientifiques de niveau recherche, publiés ou non, émanant des établissements d'enseignement et de recherche français ou étrangers, des laboratoires publics ou privés. 


\title{
gradient-index phononic crystals
}

\author{
Jinfeng Zhao ${ }^{1.2}$, Bernard Bonello ${ }^{1, *}$, Loïc Becerra ${ }^{1}$, Olga Boyko ${ }^{1}$, and Rémi Marchal ${ }^{1}$ \\ ${ }^{1}$ CNRS, UMR 7588, Institut des NanoSciences de Paris, F-75005, Paris, France \\ ${ }^{2}$ School of Aerospace Engineering and Applied Mechanics, Tongji University \\ 100 Zhangwu Road, 200092, Shanghai, China
}

\begin{abstract}
We report on the subwavelength focusing of Rayleigh waves using gradient-index (GRIN) phononic crystals (PCS) made of air holes scatters in a thick silicon substrate. The subwavelength focusing is demonstrated both in the inner and in the silicon substrate behind the GRIN PCs by using a non-contact experimental technique. In both situations, the focal zone was observed at the position in very good agreement with our theoretical predictions, at a frequency in the sound cone free of radiation into the substrate.
\end{abstract}

Over the past two decades, periodic arrays of scatters embedded in a background, known as phononic crystals (PCs), have been worth studied for reasons relating, among others, to their ability of manipulating the propagation of elastic waves and to their potential for technological applications such as filtering, guiding, acoustic diodes, etc. Investigating the conditions for complete frequency bandgaps to open in the Brillouin zone (BZ) has long been, and continues to be, a large part of the works devoted to PCs. ${ }^{1}$ Since the late 1990s, much attention has been paid to the surface acoustic wave (SAW) bandgaps in PC layer standing on a semi-infinite substrate. ${ }^{2-4}$ Later on, these periodic structures fabricated on silicon or piezoelectric substrates demonstrated their very good compatibility with the MEMS as well as their potential for applications in the wireless telecommunications and sensors. ${ }^{5,6}$ Waves guided either on the surface of a semi-infinite medium or in between the free surfaces of a plate have been studied, both theoretically ${ }^{3-8}$ and experimentally. ${ }^{9-11}$ At the same time, the shape, sizes, and arrangement of the periodic inclusions, e.g. air holes in most cases, were optimized to obtain a better confinement of the SAWs close to the surface and to avoid the radiation into the substrate, an essential prerequisite for an actual PC structure to be operational. ${ }^{10}$

In parallel to the quest for broad forbidden bands and the applications that ensue, a very active research on less conventional properties of PCs was developed. Among the most promising devices are surely the gradient-index (GRIN) PCs, which are generally formed by gradually modifying certain physical or geometrical parameters along some directions of the structure. ${ }^{12-14}$ Similarly to the GRIN optical fibers, their optical counterparts, the GRIN PCs can be used in various technological fields such as the non-destructive evaluation of materials, the energy harvesting, etc. For this purpose, the focusing of different types of waves, either in ${ }^{15-23}$ or out ${ }^{13,24}$ the homogenization regime, has been 
observed. GRIN PCs are generally engineered by drilling air holes on a silicon or a piezoelectric

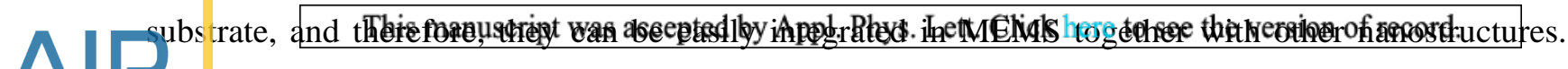
Actually, the focusing of the lowest-order flexural Lamb wave $\left(\mathrm{A}_{0}\right)$ has been reported already both Publishing numerically and experimentally in such systems. ${ }^{17,20,22}$ In contrast, the focusing of Rayleigh waves by means of a GRIN PC, has not been reported to date. Indeed, avoiding the radiation into the substrate and keeping the Rayleigh mode guided on the surface impose specific requirements on the inclusions, as already noticed in several works dealing with the opening of SAW bandgaps. ${ }^{10,11}$

In this work, we report on an in situ experimental study, conducted with the laser ultrasonic technique, of the subwavelength focusing of Rayleigh waves by GRIN acoustic lenses based onto finite-depth holey PCs.

(a)

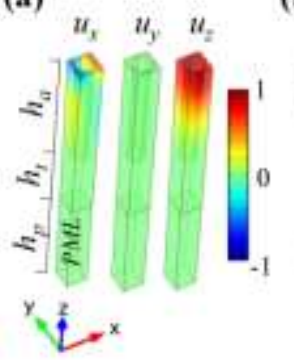

(b) 60

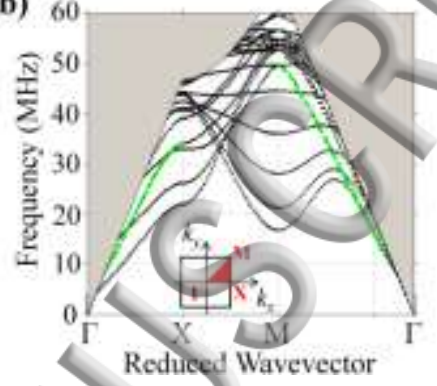

Figure 1. (a) Unit cell of the square-lattice $P C$ and normalized displacement field along $\Gamma X$ for a Rayleigh wave at $20 \mathrm{MHz}$. Perfectly matched layer (PLM) is implemented to suppress any reflection at the bottom of the unit cell. (b) Band structure (dotted lines) below the sound cone. The green lines are for the Rayleigh mode along $\Gamma X$ and $\Gamma M$.

We first have analyzed the dispersion of Rayleigh waves in PCs. To this end, we used a finite element method (FEM) to compute the band structure of the PC depicted in Fig. 1(a). The air/silicon PC is elaborated by drilling air holes in a square-lattice, over a finite depth in a silicon substrate. Below the air holes lattice lays a homogeneous silicon layer free of any inclusion ${ }^{11}$ and then, a perfectly matched layer (PML) is implemented to suppress any reflection at the bottom edge and to mimic that way the semi-infinite medium. We set the lattice constant $a=50 \mu \mathrm{m}$, the depth of the air hole $h_{a}=5 a$, the depth of silicon layer $h_{t}=3 a$, and the depth of PML $h_{p}=5 a$. We show in Fig. 1(b) the calculated band structures for air holes having a diameter $d_{a}=40 \mu \mathrm{m}$. The reduced Brillouin zone is divided into two areas by the sound line, i.e. the dispersion curve defined by the smallest phase velocity in the solid ${ }^{11}$ which is the shear-vertical mode in the present case. The shaded region above the sound line indicates an area where wave modes are radiative into the substrate while dotted curves below the sound cone are for those modes which are not. ${ }^{10,11}$ In Fig. 1(b), the Rayleigh modes along both $\Gamma \mathrm{X}$ and $\Gamma \mathrm{M}$ below the sound cone are depicted by the green dashed lines. Fig. 1(a) displays the normalized displacement field at $20 \mathrm{MHz}$ along $\Gamma X$. The displacement field polarized in the sagittal plane $x-z$ is obviously governed by the normal component $u_{z}$, and decays along the depth within about one-wavelength, in accordance with one of the main properties of the Rayleigh mode. From Fig. 1(a), one can derive 
already that neither the displacement field at $20 \mathrm{MHz}$ nor the wave vector $k_{x}$ would be affected by

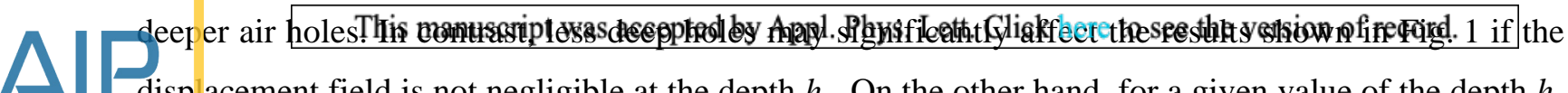
1 disp acement field is not negligible at the depth $h_{a}$. On the other hand, for a given value of the depth $h_{a}$, Publishijg g ical profiles should be obtained for frequencies higher than $20 \mathrm{MHz}$ since the penetration depth of the Rayleigh waves is smaller in that case. Two other branches appear around $20 \mathrm{MHz}$. Whatever is the filling ratio, these elastic modes are polarized in the $x-y$ plane and they do not couple with the Rayleigh mode. Moreover, they are not detected by the interferometric technique described below, which is only sensitive to the out of plane component of the displacement field.

We calculated the Rayleigh bands for different radii of the air holes, keeping unchanged all the other geometrical parameters. At a given wavenumber along $Г X$ or $\Gamma \mathrm{M}$, which are the only relevant directions for this study, both the group velocity and the frequency are all the larger that the filling ratio is small. ${ }^{22}$ Based on these trends, the strategy to design the GRIN PC consists in gradually modifying the size of the air holes in order to tune the effective refractive index $n_{\text {eff }}(y)=k_{\Gamma X} / k_{B}$ along the transverse direction ( $y$-axis). In the definition of $n_{\text {eff }}, k_{\Gamma X}$ and $k_{B}$ stand respectively for the wavenumber along $\Gamma \mathrm{X}$ in the lens and in the homogeneous silicon background on both sides of the lens. In the present case, the size of the air holes was calculated for $n_{\text {eff }}$ to fit a hyperbolic secant profile along the $y$-axis, namely $n_{\text {eff }}(y)=n_{0} \operatorname{sech}(\delta y)$. The refractive index along the central layer of our lens was $n_{0}=1.229$, the gradient coefficient was $\delta=0.080 a^{-1}$ and the total width of the lens was $D=15 a$.

(a)

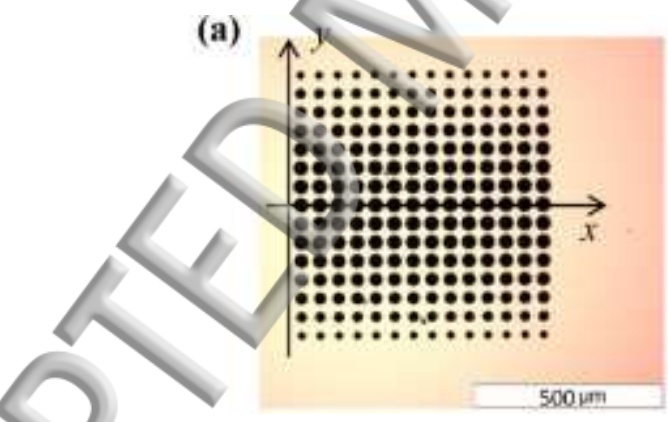

(b)

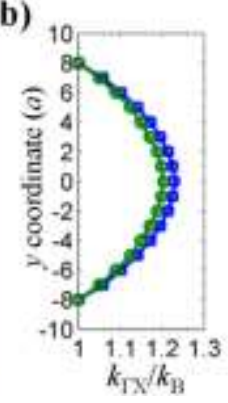

Figure 2. (a) Optical image of the sample on a silicon plate with a thickness $500 \mu \mathrm{m} .(\mathrm{b})$ designed (blue squares) and experimental (green circles) profiles of the effective refractive index.

We elaborated the samples in a clean room, on a $500 \mu \mathrm{m}$ thick silicon plate using a photolithography process followed by an isotropic dry etching. Fig. 2(a) shows an optical image of the perforated air holes on the front side of the silicon plate. The actual radii, accurately measured along all horizontal layers by optical facilities, were slightly larger than their nominal value. Moreover, upon completion of the fabrication process, the air holes were not exactly cylindrical but slightly conical with a half top angle of $\sim 1^{\circ}$, as it is often reported in literature. ${ }^{6}$ As for the depth $h_{a}$ it was measured to be larger than 
$5 a$, whatever the horizontal layer. Fig. 2(b) shows both the theoretical (square markers) and

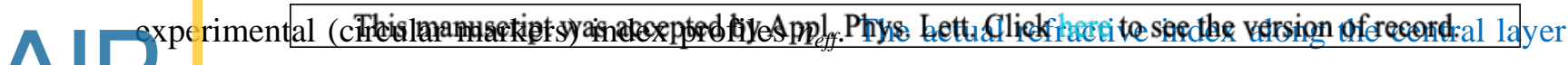
( $\left.n_{0}=1.202\right)$ and the gradient coefficient $\left(\delta=0.076 a^{-1}\right)$ both derived from the measured size of the air Publishing

and from the dispersion of the Rayleigh mode, differ from the theoretical values by $2 \%$ and $5 \%$ respectively.

It should be noted that the silicon plate was thick enough to insure that Rayleigh waves, and not Lamb waves, are actually excited at $20 \mathrm{MHz}$. Indeed, the thickness of the plate $(500 \mu \mathrm{m})$ was about twice the wavelength $(\lambda=246 \mu \mathrm{m})$ at this frequency, and as a first approximation, it can be seen as a semiinfinite medium. This is further established by the dispersion curves of the Lamb waves in the plate (not shown here). Above $\sim 16 \mathrm{MHz}$, both lower order symmetric and antisymmetric Lamb modes coincide with the Rayleigh mode.

The Rayleigh waves were excited owing to ultra-short light pulses ( 35 ps at a repetition rate of $20 \mathrm{~Hz}$ ) issued from a frequency-doubled $(532 \mathrm{~nm}) \mathrm{Nd}$ : YAG laser ${ }^{22,25}$ The light pulses went through an amplitude mask and an optical imaging system in order to create a series of about 10 bright and dark strips on the silicon plate, extending over a length of about $3 \mathrm{~mm}$ just a few millimeters in front of the sample. Emission of SAWs resulted then from photoelastic processes. The experimental configuration was set in such a way that the elastic pulses stretched along the $y$-axis, over a width $(\sim 5 \mathrm{~mm})$ much greater than the transverse width $D=15 a=750 \mu \mathrm{m}$ of the GRIN lens, insuring that plane waves impinging the sample at normal incidence are actually generated and that the light density is uniform within the photoelastic source. Note also that the portions of the elastic beam above and below the lens (i.e. $|y|>D)$, do not participate in the focusing process.

The normal displacements were measured using a He-Ne laser $(632.8 \mathrm{~nm})$ as the light source of a Michelson interferometer, with the sample surface acting as one of the mirrors of the interferometer and an actively stabilized mirror as the other one. The interference pattern was collected on a highspeed photodetector and then digitized at $500 \mathrm{MS} \cdot \mathrm{s}^{-1}$ by a digital oscilloscope. A very good signal/noise ratio is obtained after averaging a few hundreds of scans. This non-contact technique allowed us to record out-of-plane displacements $u_{z}$ as small as a few pm, at any place inside or outside the GRIN PC with a precision close to $1 \mu \mathrm{m}$. Most of the experiments described here were done at a carrier frequency centered on $19.8 \mathrm{MHz}$, very close to the targeted frequency of $20 \mathrm{MHz}$, and a bandwidth of $2 \mathrm{MHz}$. The wavelength measured on the silicon plate was $250 \mu \mathrm{m}$, in very good agreement with the theoretical value of $246 \mu \mathrm{m}$ derived from the velocity of the Rayleigh wave, namely $4938 \mathrm{~m} / \mathrm{s}$. 


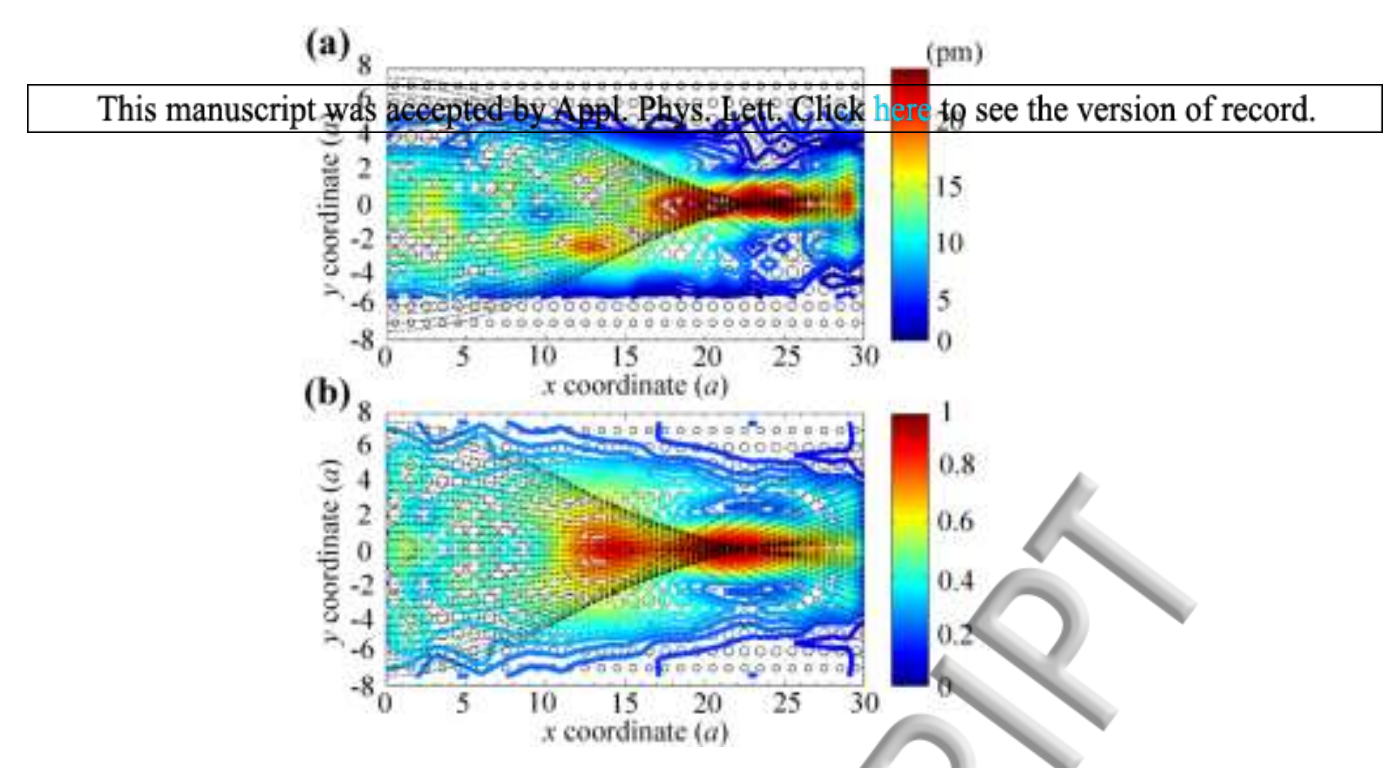

Figure 3. (a) Experimental and (b) numerical maxima of $u_{z}$ in the acoustic lens with 30 columns of air inclusions. Ray trajectories (thin dashed lines) were obtained by using the experimental and the numerical wave numbers, respectively.

We first measured the normal displacement inside an acoustical lens featuring 30 unit-cells along the $x$-axis (i.e. the total length is 30a). We show in Fig. 3(a) the map of the measured maxima of $u_{z}$ in the acoustic lens. The overall maximum $u_{z}$ of $25.9 \mathrm{pm}$ was found at the position $x=25 a$ on the central layer, and not at the position $x=20.7 a$ as predicted by the relation $\pi / 2 \delta$ derived from a basic ray trajectory theory. ${ }^{17,26}$ This discrepancy is well explained when one takes account of the actual shape of the equifrequency contours (EFC's) along each line of inclusions to calculate the ray trajectories, as shown by the computed rays drawn as black dashed lines in Fig. 3(a). The formalism consists in calculating the angle between the group velocity (normal to the EFC) and the phase velocity (parallel to the wave vector) at any point of the EFC. ${ }^{25}$ The acoustic rays do not converge to a single point, as it should be for a planar GRIN lens with hyperbolic secant profile, which is free of aberrations. ${ }^{26}$ Instead, the computed focusing zone extends on the central layer over the interval $x=[22 a, 25 a]$, in very good agreement with the experimental results at $x=[22.7 a, 25.2 a]$ where the amplitude $u_{z}$ takes a value larger than $95 \%$ of the maximum value $25.9 \mathrm{pm}$.

We have also simulated the achievements of the lens using Comsol FEM. For these computations, we set the thickness of the silicon substrate to a value large enough to prevent any reflection at the boundaries of the plate. Fig. 3(b) shows the maxima of $u_{z}$ normalized by the overall maximum value, found at the position $x=21 a$, together with the ray trajectories. The latter delineate the focus area $(95 \%$ of the maximum amplitude) at about $x=[21 a, 24 a]$, in very good agreement with the position $(95 \%$ of the maximum amplitude) determined by the simulations to lie at $x=[20.5 a, 22.7 a]$. It is worth noting that the rays drawn in Figs. 3(a) and 3(b) were derived from the EFCs which were computed by accounting for the actual conical-shaped holes and the cylindrical-shaped holes respectively. This allowed quantifying the consequences of a small geometrical irregularity of the inclusions (around $1^{\circ}$ 
in the present case). The experiments, the simulations and the theory lead all to results on the

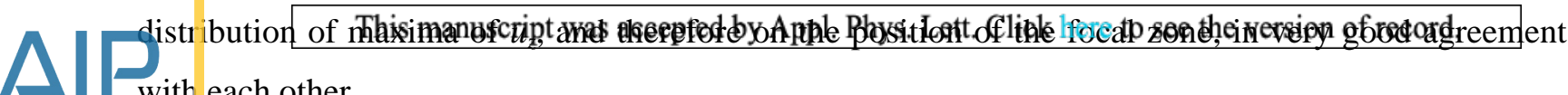
with each other.

Publishing

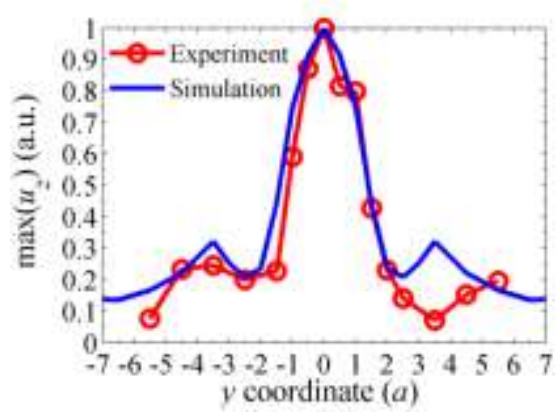

Figure 4. Experimental (circles) and numerical (full line) normalized profiles of the maxima of $u_{z}$ along the transverse direction, at the focus

The agreement between the transverse profiles of the maxima of $u_{z}$ at the focus, obtained either experimentally (red dotted line) or numerically (blue line) is very good (Fig. 4). The full width at half maximum (FWHM) normalized to the wavelength in the central layer $\lambda_{\min }$, is $0.61 \lambda_{\min }(2.53 a)$ when measured experimentally and $0.70 \lambda_{\min }(2.81 a)$ if derived from the simulations. The good performances of our lens can be ascribed to the large gradient coefficient $\delta$ that leads to a large curvature for the marginal rays and in turn to an output numerical aperture (defined as the sine of the half top angle ${ }^{26}$ ) estimated to $\mathrm{NA}=0.28$. Moreover, there are few geometrical aberrations at the focus since the velocity of the Rayleigh waves in the lens depends little on the direction of propagation (the maximum value of the ratio $k_{\Gamma M} / k_{\Gamma X}$ is 1.06). To conclude this section, it should be mentionned that an even smaller FWHM would require a larger maximum value of the transverse wavenumber $k_{\mathrm{y}}$ at the focus (or equivalently a larger gradient eoefficient $\delta$ ), at the expense of larger geometrical aberrations along the central layers. ${ }^{25}$

Concentrating the elastic energy over a spot inside the lens may be advantageous in certain specific circumstances (e.g. selectively exciting a cavity mode) but more generally an acoustical lens is aimed at imaging an object on a plane behind it. We thus have elaborated a shorter acoustical lens featuring 14 unit cells along the $x$-axis and all other parameters kept unchanged. We repeated the laser-based experiments to map the maximum value of the normal component $\left|u_{z}\right|$. The displacement field displayed in Fig. 5(a), features a peak of about $19.6 \mathrm{pm}$, at $x=17.5 \mathrm{a}$ on the central layer, to be compared with the maximum amplitude $25.9 \mathrm{pm}$ measured inside the lens; the focal zone stretches over the interval $x=[16.7 a, 18.2 a]$. The ray trajectories (fine dashed lines in Fig. 5(a)), which are straight lines in the silicon substrate upon refraction at the interface, well account both for the position of the focus and for the geometrical aberrations resulting from the anisotropy along each line of inclusions. 
(a) (b)

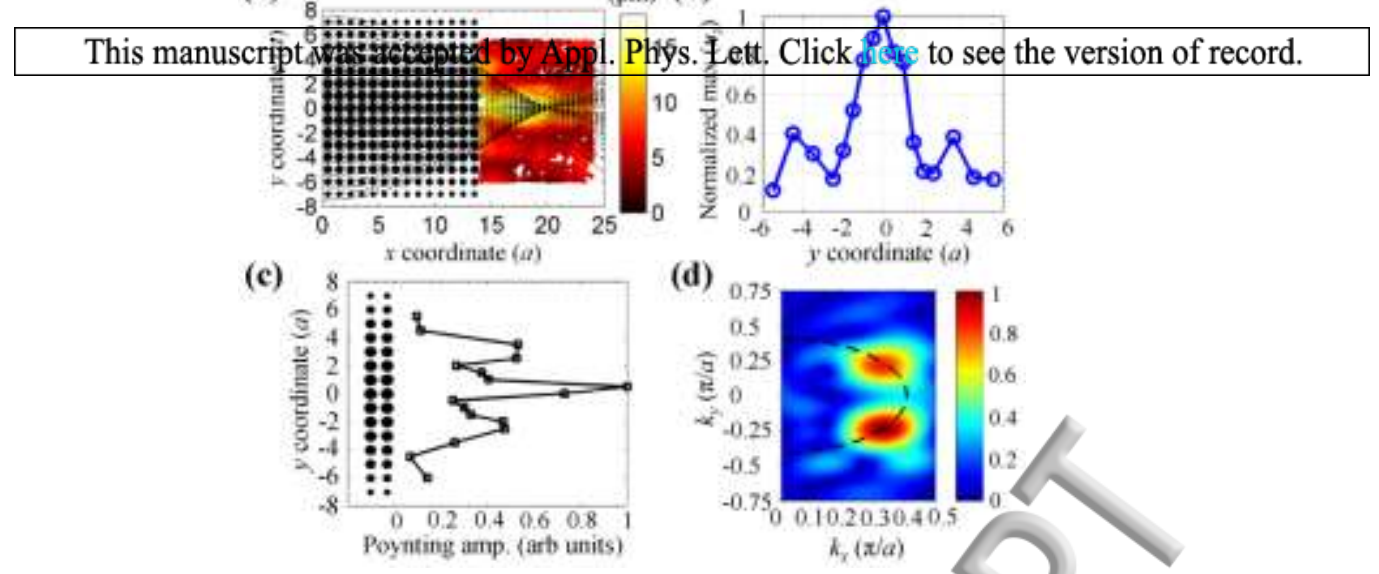

Figure 5. (a) Experimental distribution of displacement field behind a lens with 14 columns of air inclusions. (b) Amplitude along y-axis at the focus. (c) Normalized Poynting vector along the interface. (d) $2 D$ Fourier transform of the displacement field when the amplitude is maximum at the focus. The dashed line is for the EFC at $20 \mathrm{MHz}$.

Fig. 5(b) shows the transverse profile measured at the focus: the FWHM is $2.87 a$. When reported to the wavelength in the background $\left(\lambda_{\mathrm{B}}=4.91 a\right)$, the FWHM is equal to $0.58 \lambda_{\mathrm{B}}$, i.e. slightly better than the inner focusing resolution $0.61 \lambda_{\min }$. Clearly, this should be-ascribed to the reference wavelength which is larger in the substrate than it is in the lens. It is also important to notice that the elastic energy is not evenly distributed along the interface. Qualitatively, this can be seen from the density of rays drawn in Fig. 5(a) which is not uniform on the silicon plate downstream the lens but shows a maximum on the edges of the beam. A quantitative approach requires calculating the components of the Poynting vector whose module is proportional to the quantity $P=\left[\sum_{i=1}^{2}\left(C_{i j k l} u_{j} u_{k} x_{l}\right)^{2}\right]^{1 / 2}$ where $C$ stands for the elastic constants of silicon. An estimate of $P$ along the interface can be derived from the measurement of $u_{z}$. Actually, close to the free surface, the in-plane and the out-of-plane components of the Rayleigh wave are in a constant ratio which in silicon is close to $u_{z} / u_{x} \approx 1.18$. ${ }^{27}$ We used this value to derive $P$ against $y$ normalized to unity from our experimental data (Fig. 5(c)). In addition to the sharp peak at $y=0$, the elastic energy is mainly concentrated around $y= \pm 3 a$, at a position where the component $k_{y}$ of the wave vector is equal to $\mp 0.24 \pi / a$. We then have analyzed the dispersion of the waves in the background. To this end, we have computed the 2D Fourier transform of the displacement field recorded on a large area behind the lens at the moment when the amplitude at the focus is maximum. The result displayed in Fig. 5(d), clearly shows two main spots centered at $k_{y}= \pm 0.24 \pi / a$ well on the EFC of the Rayleigh wave at $20 \mathrm{MHz}$ (dashed line), in excellent agreement with the distribution of elastic energy on the front wave just behind the interface. 
In summary, we demonstrated the subwavelength focusing of a Rayleigh wave at a frequency in the

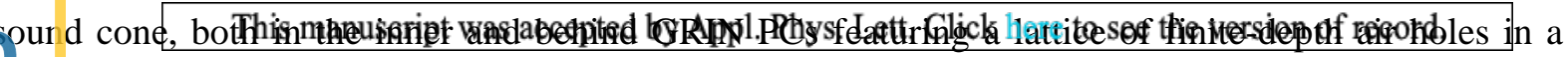
1 thick silicon plate. By using the non-contact laser ultrasonic technique to measure the out-of-plane Publishing acement, we have been able to demonstrate FWHM as small as $0.63 \lambda_{\text {min }}$, in good agreement with both the analytical and the numerical counterparts. In a sample shorter than the focal length, we obtained experimentally a spot behind the acoustic lens featuring FWHM of $0.58 \lambda_{\mathrm{B}}$, as expected from the computation of the ray trajectories.

\section{References}

*Corresponding author: bernard.bonello@insp.jussieu.fr

${ }^{1}$ M. S. Kushwaha, P. Halevi, L. Dobrzynski, and B. Djafari-Rouhani, Phys. Rev. Lett. 71, 2022 (1993).

${ }^{2}$ Y. Tanaka and S.-i. Tamura, Phys. Rev. B 60, 13294 (1999).

${ }^{3}$ M. Torres, F. R. Montero de Espinosa, D. García-Pablos, and N. García, Phys. Rev.Lett. 82, 3054 (1999).

${ }^{4}$ S. Benchabane, A. Khelif, J.-Y. Rauch, L. Robert, and V. Laude, Phys. Rev.E 73, 065601 (2006).

${ }^{5}$ V. Laude, M. Wilm, S. Benchabane, and A. Khelif, Phys. Rev. E71, 036607 (2005).

${ }^{6}$ T.-T. Wu, L.-C. Wu, and Z.-G. Huang, J. Appl. Phys. 97, 094916 (2005).

${ }^{7}$ J.-H. Sun and T.-T. Wu, Phys. Rev. B 74, 174305 (2006).

${ }^{8}$ Y.-Z. Wang, F.-M. Li, W.-H. Huang, and Y.-S. Wang, J. Mech. Phys. Solids 56, 1578 (2008).

${ }^{9}$ D. Yudistira, S. Benchabane, D. Janner, and V. Pruneri, Appl. Phys. Lett. 95, 052901 (2009).

${ }^{10}$ S. Benchabane, O. Gaiffe, G. Ulliac, R. Salut, Y. Achaoui, and V. Laude, Appl. Phys. Lett. 98, 171908 (2011).

${ }^{11}$ D. Yudistira, Y. Pennec, B. Djafari-Rouhani, S. Dupont, and V. Laude, Appl. Phys. Lett. 100, 061912 (2012).

${ }^{12}$ D. Torrent and J. Sánchez-Dehesa, New J. Phys. 9, 323 (2007).

${ }^{13}$ K. Deng, Y. Ding, Z. He, Z. Liu, H. Zhao, J. Shi, and Z. Liu, J. Phys. D: Appl. Phys. 42, 185505 (2009).

${ }^{14}$ S.-C. Lin, T. J. Huang, J.-H. Sun, and T.-T. Wu, Phys.Rev. B 79, 094302 (2009).

${ }^{15}$ T. P. Martin, M. Nicholas, G.J. Orris, L.-W. Cai, D. Torrent, and J. Sánchez-Dehesa, Appl. Phys. Lett. 97, 113503 (2010).

${ }^{16}$ D. Torrent, Y. Pennec, and B. Djafari-Rouhani, Phys.Rev. B 90, 104110 (2014).

${ }^{17}$ T.-T. Wu, Y.-T. Chen, J.-H. Sun, S.-C. S. Lin, and T. J. Huang, Appl. Phys. Lett. 98, 171911 (2011).

${ }^{18}$ L. Zigoneanu, B.-I. Popa, and S. A. Cummer, Phys. Rev. B 84, 024305 (2011).

${ }^{19}$ T. M. Chang, G. Dupont, S. Enoch, and S. Guenneau, New J. Phys. 14, 035011 (2012).

${ }^{20}$ M.-J. Chiou, Y.-C. Lin, T. Ono, M. Esashi, S.-L. Yeh, and T.-T. Wu, Ultrasonics 54, 1984 (2014).

${ }^{21}$ Y. Ye, M. Ke, Y. Li, T. Wang, and Z. Liu, J. Appl. Phys. 114, 154504 (2013).

${ }^{22}$ J. Zhao, R. Marchal, B. Bonello, and O. Boyko, Appl. Phys. Lett. 101, 261905 (2012).

${ }^{23}$ Y. Jin, D. Torrent, Y. Pennec, Y. Pan, and B. Djafari-Rouhani, Sci. Rep. 6, 24437 (2016)

${ }^{24}$ S. Peng, Z. He, H. Jia, A. Zhang, C. Qiu, M. ke, and Z. Liu, Appl. Phys. Lett. 96, 263502 (2010).

25. Zhao, B. Bonello, R. Marchal, and O. Boyko, New J. Phys. 16, 063031 (2014).

${ }^{26}$ C. Gomez-Reino, M. V. Perez, and C. Bao, Gradient-Index Optics: Fundamentals and Applications (Springer, Berlin, 2002).

${ }^{27}$ D. Royer and E. Dieulesaint, in Elastic Waves in Solids, 2000 Ed. Springer-Verlag Berlin Heidelberg. 
(a)
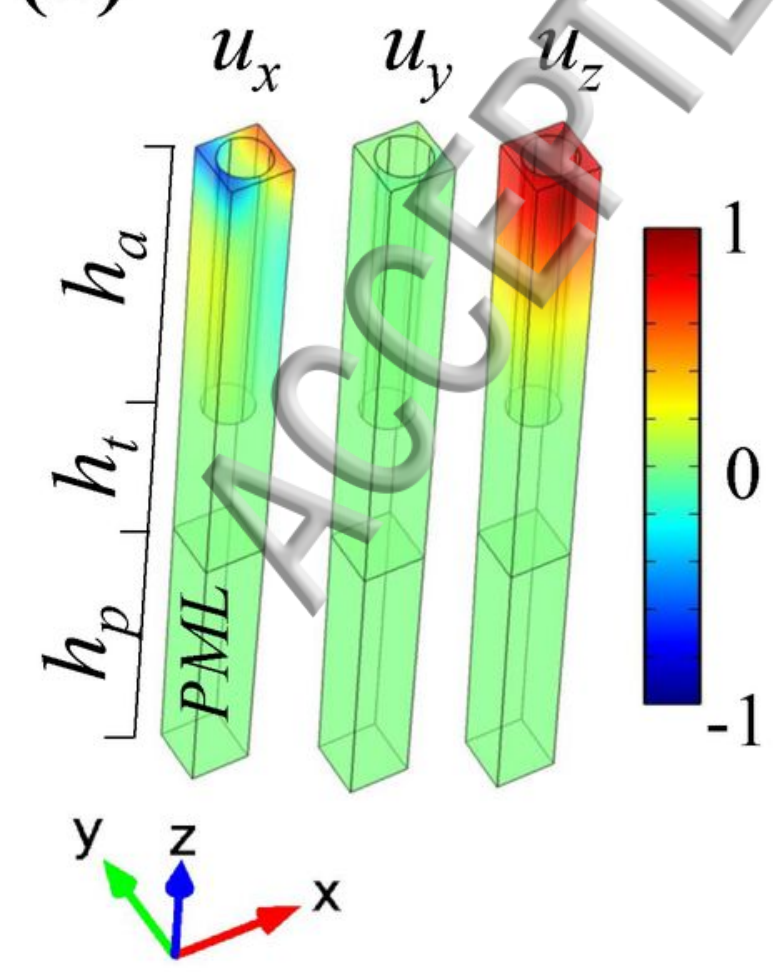

(b) 60

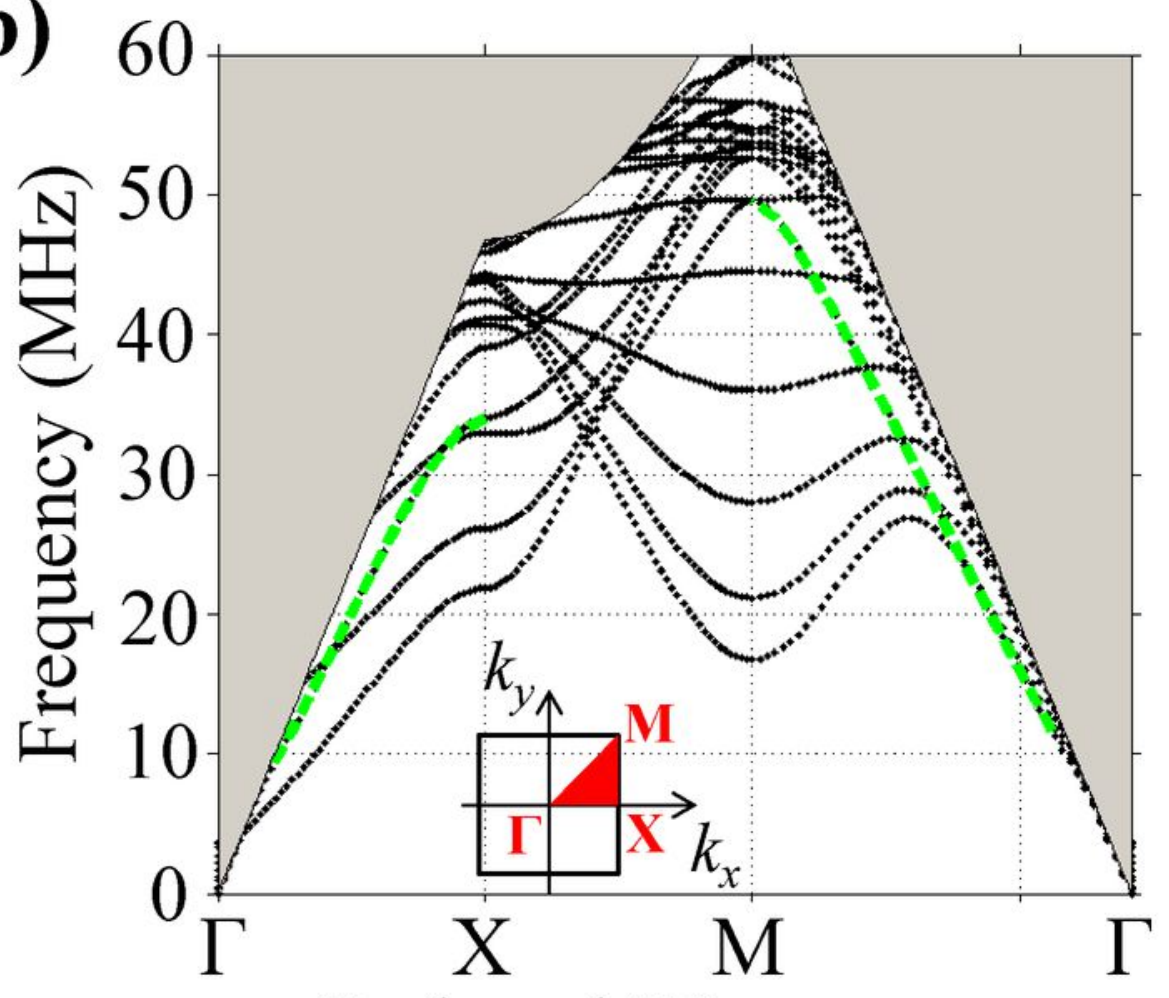

Reduced Wavevector 


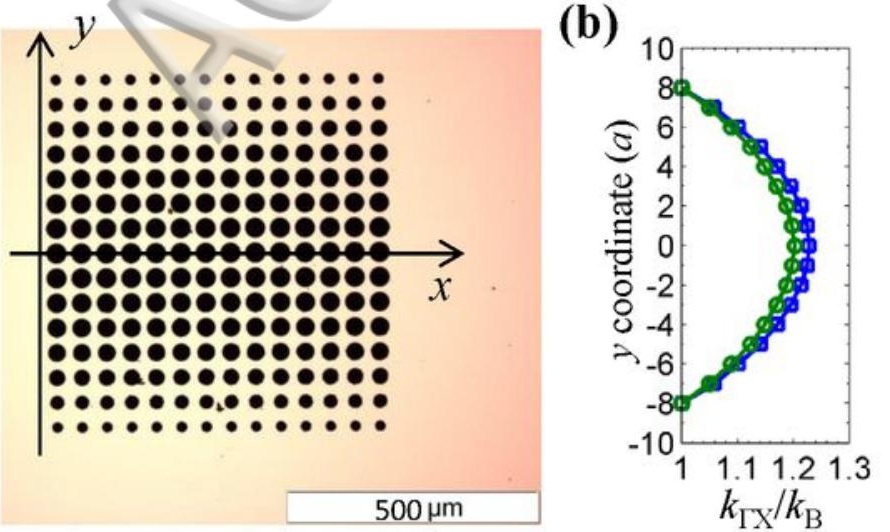


8

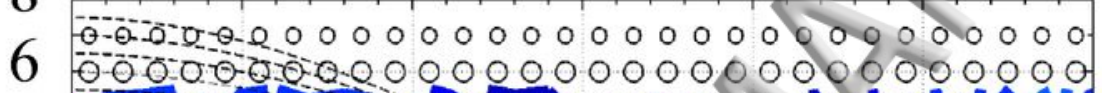

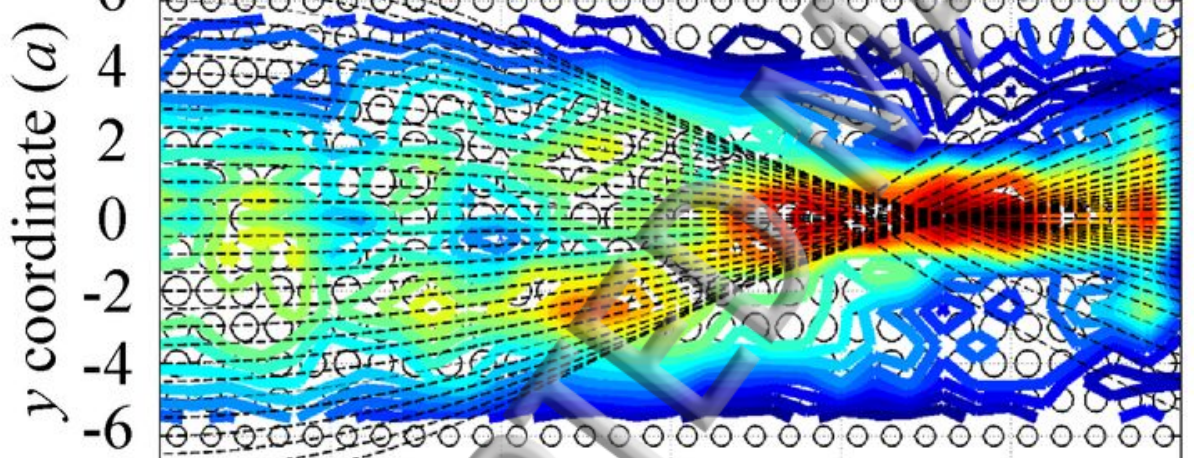

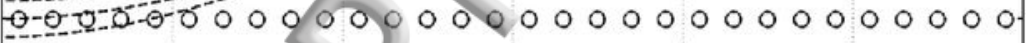

$-8$

$\begin{array}{lllllll}1 & & 1 & 1 & 1 & 1 & 1 \\ & 5 & 10 & 15 & 20 & 25 & 30\end{array}$

(pm)

$x$ coordinate $(a)$

(b)

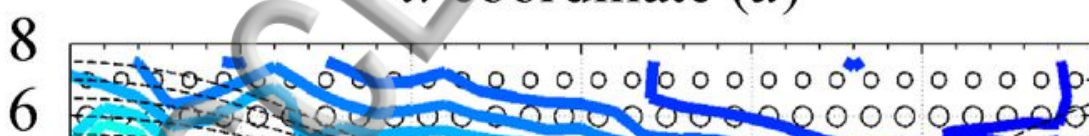

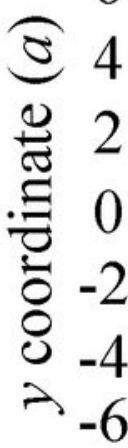

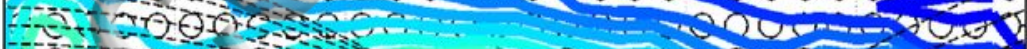

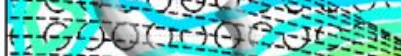

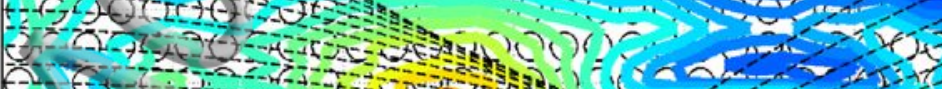

6. $E$ -

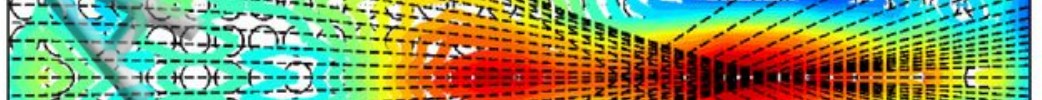

E-

(1)

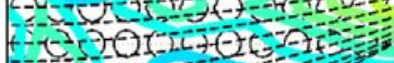

-

政?

, $, 0,0,2$

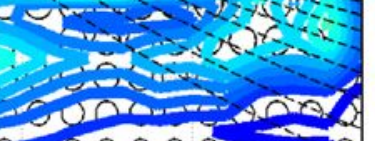

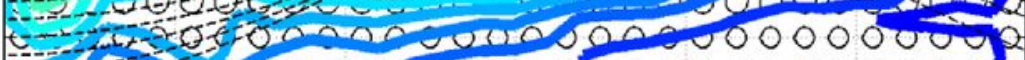

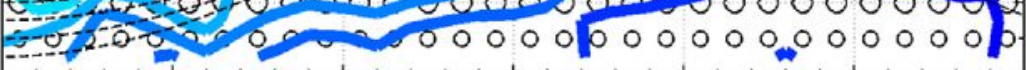

$-8$

0

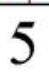

10

15

20

25

30

0.8

0.6

0.4

0.2

$x$ coordinate $(a)$ 


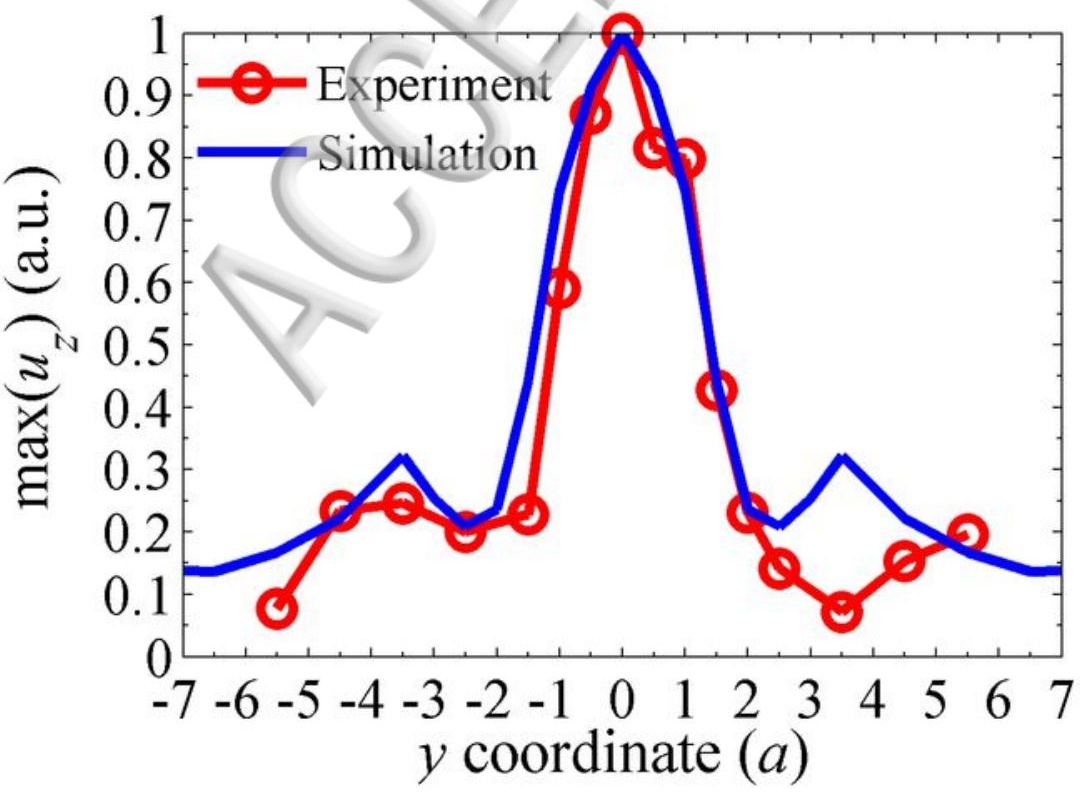




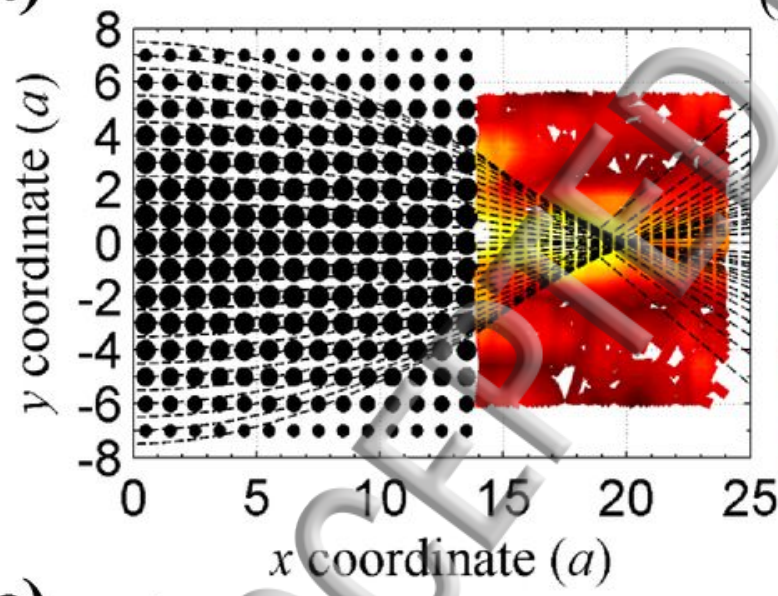

(pm) (b)

(c)

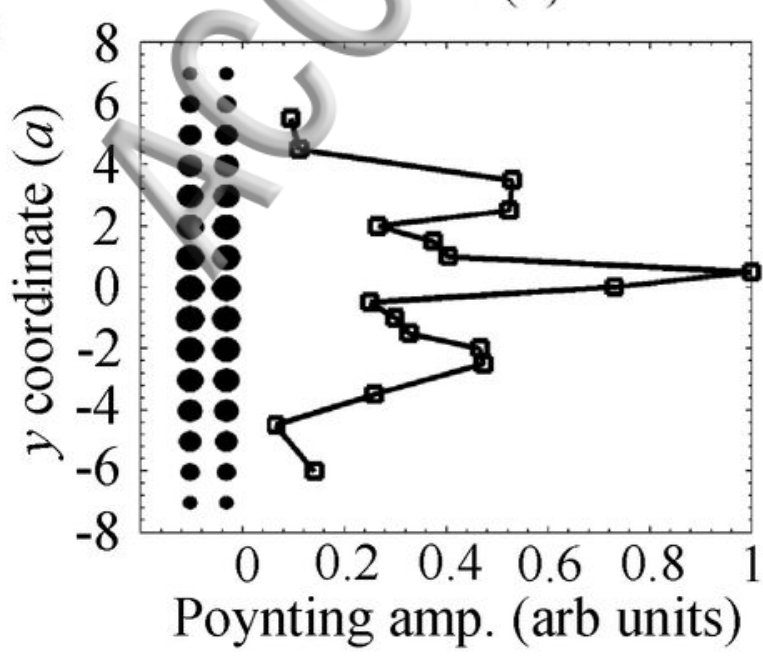

(d)

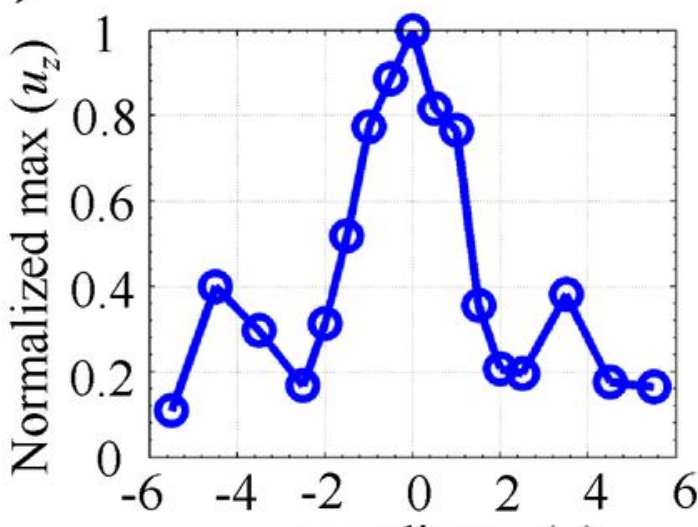

$y$ coordinate $(a)$

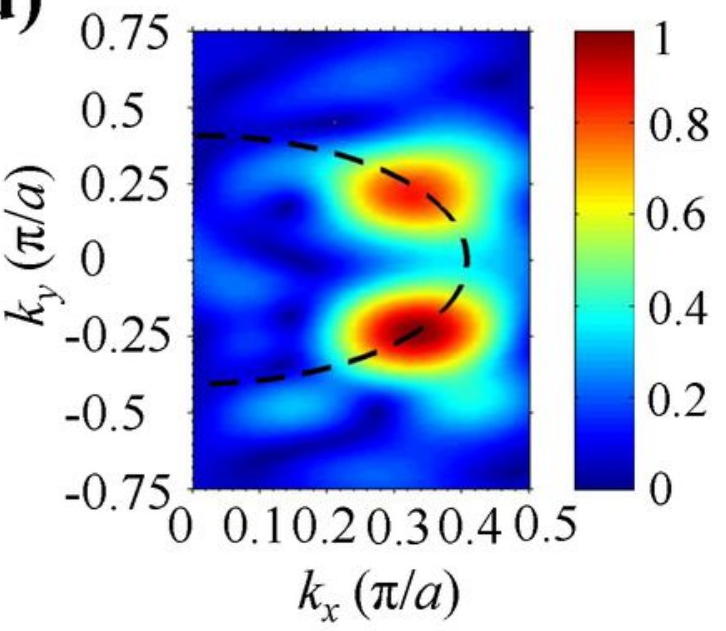

